\title{
Investigating the recording and accuracy of fluid balance monitoring in critically ill patients
}

\author{
A Diacon, ${ }^{1,2}$ MCur; J Bell, ${ }^{1,3}$ MCur, BCur, PGDN \\ ' Division of Nursing, Faculty of Medicine and Health Sciences, Stellenbosch University, Cape Town, South Africa \\ ${ }^{2}$ TASK Applied Science, Karl Bremer Hospital, Bellville, Cape Town, South Africa \\ ${ }^{3}$ Department of Nursing Science, School of Clinical Care Sciences, Faculty of Health Sciences, Nelson Mandela Metropolitan University, \\ Port Elizabeth, South Africa
}

Corresponding author: A Diacon (adiacon@mac.com)

Background. The accurate assessment of fluid balance data collected during physical assessment as well as during monitoring and record-keeping forms an essential part of the baseline patient information that guides medical and nursing interventions aimed at achieving physiological stability in patients. An informal audit of 24-hour fluid balance records in a local intensive care unit (ICU) showed that seven out of ten fluid balance calculations were incorrect.

Objective. To identify and describe current clinical nursing practice in fluid balance monitoring and measurement accuracy in ICUs, conducted as part of a broader study in partial fulfilment of a Master of Nursing degree.

Methods. A quantitative approach utilising a descriptive, exploratory study design was applied. An audit of 103 ICU records was conducted to establish the current practices and accuracy in recording of fluid balance monitoring. Data were collected using a purpose-designed tool based on relevant literature and practice experience.

Results. Of the original recorded fluid balance calculations, $79 \%$ deviated by more than $50 \mathrm{~mL}$ from the audited calculations. Furthermore, a significant relationship was shown between inaccurate fluid balance calculation and administration of diuretics $(p=0.01)$.

Conclusion. The majority of fluid balance records were incorrectly calculated.

S Afr J Crit Care 2014;30(2):55-57. DOI:10.7196/SAJCC.193

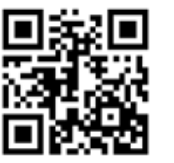

Maintaining a balance between fluid intake and output plays an important role in the management of a critically ill patient. The accurate assessment of the fluid balance data collected during physical assessment as well as during monitoring activities and record-keeping forms an essential part of the baseline patient information that guides medical and nursing interventions to achieve physiological stability in a patient. Changes in a critically ill patient's fluid balance can complicate the patient's clinical condition. It is, therefore, necessary that fluid balance parameters are accurately monitored and recorded for all patients in intensive care units (ICUs) ${ }^{[1]}$

A daily observation sheet is used to record all vital signs, nursing interventions, medical procedures and the fluid balance for each 24-h period of a day. The fluid balance record comprises records of the intake and output of fluids by a patient over a 24-h period. The difference between the volumes is calculated to provide the 24-h fluid balance. ${ }^{[2]}$ The monitoring of a patient's fluid balance is of great importance in understanding and managing a patient's clinical status and, as such, accurate monitoring and recording of fluid balance data plays an essential role in patient care management. ${ }^{[3]}$

Several studies have considered the relationship between fluid imbalances and patient outcomes in critical care. The Sepsis Occurrence in Acutely III Patients (SOAP) study by Vincent et al., ${ }^{[4]}$ conducted across 198 ICUs in Europe in 2002, determined that a positive fluid balance is a strong prognostic factor for death in critically ill patients. Similarly, research by Alsous et al., ${ }^{[5]}$ Boyd et al. ${ }^{[6]}$ and Payen et $a l^{[7]}$ concluded that a more positive fluid balance is associated with an increased risk of mortality in patients with septic shock or acute renal failure. Furthermore, Rosenberg et al. ${ }^{[8]}$ determined that a cumulative negative fluid balance in patients with acute lung injury is associated with lower mortality. The conclusions offered by these studies require that monitoring and recording of fluid balance data must be complete and accurate, with assessment of a patient's fluid balance being recognised as an important component of nursing any critically ill patient.

In South Africa (SA), the practice of a registered nurse is regulated by the Scope of Practice drawn up by the SA Nursing Council. ${ }^{[9]}$ Chapter 2, section 2(i) of these regulations identifies that fluid balance monitoring is part of the scope of practice of a registered nurse. Therefore, a registered nurse working in a critical care environment is responsible and accountable for the accurate recording and calculation of fluid balance when caring for and managing a critically ill patient. Managing a patient's fluid balance is as equally important as carrying out any other patient care activity for the critically ill, such as administering a medication prescription or providing nutrition. ${ }^{[2]}$

Fluid balance management in ICU patients is complex. Monitoring and measurement of fluid balance requires close attention to ensure that current methods are applied accurately and consistently to provide the most complete data, upon which patient management decisions can be based.

Based on practice experience and underpinned by an informal audit of 24-h fluid balance charts in a local ICU, where seven out of ten calculated totals were incorrect, the research question posed was: What are the current practices of registered nurses in ICUs with regard to fluid balance monitoring? 


\section{Methods}

A quantitative approach utilising an exploratory, descriptive study design was applied. The study was conducted in ICUs across three purposively selected hospitals of one private sector hospital group. The ICUs of these hospitals were similar in terms of their patient admission profiles, with the same nursing documentation and policies applied at all three hospitals.

An audit tool was developed from relevant literature and clinical experience to assess particular aspects of the sampled fluid balance records. Two critical care nurse experts evaluated the content and face validity of the audit tool; no changes were required. A pretest of the audit tool was conducted at one additional ICU of the same hospital group to determine the accuracy and relevance of the measurements; no changes were required. The pretest data were not included in the study data. A statistician determined the tool to be appropriate and adequate for data collection and analysis purposes.

Ethical approval for the study was obtained from the Human Research Ethics Committee at the Faculty of Medicine and Health Sciences, Stellenbosch University, as well as the relevant committee of the hospital group.

The population for this study was critical care patient records. The study sample was drawn from fluid balance records according to the following inclusion criteria:

- Nursing records of admissions to ICUs for the first $48 \mathrm{~h}$ of the patient's stay, from 1 July to 31 December 2011

- Patients over the age of 18 years as per the definition of an adult in the Children's Act No. 38 of $2005^{[10]}$

- Patients classified as 'intensive care': activity 1 or 2 on the patient classification system of this hospital group. This classification was used by the doctor to determine financial charges to the patient. No written policy regarding this classification was available from the hospitals.

A simple random sampling technique was implemented to select patient records for the audit: all the admission numbers of patients meeting the inclusion criteria were identified through the hospital information system and admission record book of the ICU. The patient record file that was connected with every third patient admission number was drawn until the required sample was achieved. The sample size was calculated to ensure adequate precision in population estimates, using $95 \%$ confidence intervals ( $\mathrm{Cls}$ ). A sample size of 80 fluid balance records would have resulted in $6 \%$ precision in the $95 \% \mathrm{Cl}$ width, assuming a $10 \%$ error rate in the calculation of the fluid balance. This was well within the accepted precision of between $5 \%$ and $10 \%$. A sample size of $N=103$ was selected and divided specifically among the various units under the guidance of the statistician (Table 1). Descriptive statistics were recorded and the Mann-Whitney U-test was used to test associations between recorded variables and fluid balance calculation accuracy.

Data were recorded on the study audit tool by the researcher and a field worker together in the three hospitals. The fluid balance calculation recorded in each patient record for a 24-h period during the first $48 \mathrm{~h}$ of a patient's stay was noted on the audit tool. A control calculation of each recorded fluid balance total was done by the researcher and verified by the field worker. These audited calculations were recorded in the audit tool. The deviation between the original calculations and the audited calculations was determined and recorded.

In addition to the fluid balance calculation, baseline vital sign data, modes of fluid output (e.g. diarrhoea), specific data regarding the administration of blood products, and the number of continuous intravenous infusions were recorded on the audit tool.

\section{Results \\ 24-h calculated fluid balance totals}

The original recorded 24-h fluid balance total was compared with the audited fluid balance total performed by the researcher and field worker. The difference in calculation was referred to as the deviation in fluid balance calculation, and is presented in Table 2 for descriptive reasons.

In the audit of 103 fluid balance documents, a total of $71(68.9 \%)$ recorded calculated fluid balance totals were within a $500 \mathrm{~mL}$ deviation from the fluid balance calculated by the researcher. Fourteen recorded calculations (13.5\%) were found

Table 1. Sampling framework

\begin{tabular}{llll}
\hline Hospital & Intensive-care beds, $\boldsymbol{n}$ & $\begin{array}{l}\text { Admissions: } \\
\text { July - December 2011, } \boldsymbol{n}\end{array}$ & Records sampled, $\boldsymbol{n}$ \\
\hline A & 26 & 1020 & 34 \\
B & 28 & 1027 & 34 \\
C & 38 & 1022 & 35 \\
D & 12 & 300 & Pilot study
\end{tabular}

Table 2. Deviation in fluid balance $(N=103)$

\begin{tabular}{|c|c|c|c|c|c|c|c|}
\hline Calculated deviation & $\begin{array}{l}\text { Overall } \\
0-3706\end{array}$ & $0-50$ & $51-500$ & $501-1000$ & $1001-2000$ & $>2001$ & No record \\
\hline$n$ & 98 & 22 & 49 & 14 & 7 & 6 & 5 \\
\hline Percentage & 95.1 & 21 & 48 & 13.5 & 6.8 & 5.8 & 4.9 \\
\hline Median deviation (mL) & 167 & 20 & 146 & 754 & 1249 & 3310 & - \\
\hline Mean deviation (mL) & 493 & 21 & 184 & 754 & 1371 & 3116 & - \\
\hline Range (mL) & $0-3706$ & $0-46$ & $61-463$ & $501-984$ & $1008-1928$ & $2260-3706$ & - \\
\hline
\end{tabular}


Table 3. Comparison of accurate and inaccurate fluid calculation

\begin{tabular}{lccl}
\hline \multirow{2}{*}{ Variable } & \multicolumn{2}{c}{ Inaccurate fluid calculation, median (IQR) } & \\
\cline { 2 - 3 } & Yes & No & -value \\
\hline Received blood products & $180.5(60-1312)$ & $167(61-530)$ & 0.95 \\
CVP measured & $202.5(90-764)$ & $119(41-320)$ & 0.09 \\
Matched doctor's prescription & $155(60-530)$ & $201(63-708)$ & 0.61 \\
Diuretic administered & $279(102-996)$ & $106(46-350)$ & 0.01 \\
Received >2 intravenous drugs & $257(75-708)$ & $138(60-435)$ & 0.16 \\
IQR $=$ interquartile range; CVP $=$ central venous pressure. & &
\end{tabular}

to deviate between $500 \mathrm{~mL}$ and $1000 \mathrm{~mL}$, while seven recorded calculations $(6.8 \%)$ were found to deviate between $1000 \mathrm{~mL}$ and $2000 \mathrm{~mL}$. Six recorded calculations $(5.8 \%)$ were found to have a deviation of $>2000 \mathrm{~mL}$.

There was a significant association between the administration of diuretics and inaccurate fluid balance calculation $(p=0.01)$, but there was no association between other variables and the outcome of interest (Table 3 ).

\section{Discussion}

The definition of a net positive fluid balance as a volume $\geq 500 \mathrm{~mL}$ used in the study by Alsous et $a l .{ }^{[5]}$ was applied in this study. Of great concern were the 27/103 documents, more than $25 \%$ of the sample, with a deviation of $>500 \mathrm{~mL}$ between the recorded calculation and the control calculation. Equally of concern were the five patient records where no fluid balance calculation was available at all. These findings represent a risk for the critically ill patient when one considers the findings of previous studies related to positive fluid balance and patient mortality. ${ }^{[4-8]}$ The findings of this study showed that fluid balance calculation is not treated as a priority in the nursing management of a critically ill patient. The incorrect calculation of fluid balance means that every patient management decision utilising these fluid balance data was influenced by inaccurate information. Perren et al. ${ }^{[1]}$ performed a similar study in Switzerland and expressed their concern about the accuracy of fluid balances in critically ill patients. ${ }^{[11]}$

Additionally, the significant association between inaccurate fluid balance calculation and diuretic administration $(p=0.01)$ suggests that when diuretics are administered, there is a higher chance of the calculated fluid balance being incorrect. This finding supports the researcher's concern that a careful and accurate approach to fluid balance does not enjoy high priority in managing critically ill patients in this context. Diuretic therapy is a commonly prescribed therapeutic modality; in this study, $38.8 \%$ (40/103) of critically ill patients had diuretics recorded as being administered during the first $48 \mathrm{~h}$ of their admission. Inaccurate fluid balance data may result in inappropriate application of diuretic therapy, resulting in fluid imbalances that affect the haemodynamic stability of patients.

The findings of this study are limited by the focus on one hospital group and may be regarded as a pilot study for further development.

\section{Conclusions}

In this study, the majority of audited 24-h fluid balance calculations were shown to be incorrect; $79 \%(81 / 103)$ of the original recorded fluid balance calculations deviated by $>50 \mathrm{~mL}$ from the audited calculation. The accuracy of the 24-h balance calculated is questionable, with only $21 \%$ of the original fluid balance totals deviating by $\leq 50 \mathrm{~mL}$ from the audit calculations. This is of great concern. Several studies ${ }^{[4-8]}$ have noted a relationship between fluid imbalance and mortality in critically ill patients. The findings indicate that treatment decisions are often based on inaccurate fluid balance information, which may lead to negative consequences for the patient.

A significant association was shown between the administration of diuretics and inaccurate 24-h fluid balance calculations. With diuretics prescribed specifically to manage fluid imbalance, this finding indicates that the accuracy of the calculated fluid balance must be confirmed prior to diuretics being prescribed or administered.
Within the context of limited resources, any clinical recommendations must be realistic and practical. One suggested example is instituting a system of checking fluid balance calculations at specific intervals, such as during patient handover at shift change, during the patient assessment process or during patient management discussions. Awareness around the potential consequences of calculation errors must be reinforced during patient discussions and continuing education sessions.

The requirement to provide accurate, correct fluid balance monitoring and recording as part of the patient's vital sign data must be established as a fundamental standard of practice for every nurse practising in an ICU. Regular outcomedriven audits will assist in identifying where and when errors occur, allowing for specific interventions to be designed and implemented.

Further studies may assist in refining the particular challenges of accurate fluid balance recording, for instance cumulative fluid balance over more than $24 \mathrm{~h}$.

\section{References}

1. Culleiton AL, Simko LC. Keeping electrolytes and fluids in balance. Nursing2013 Critical Care 2011;6(2):30-35. [http:// balance. Nursing2013 Critical Care 2011;6(2):30-35.
dx.doi.org/10.1097/01:CCN.0000394395.67904.4d]

2. Scales K, Pilsworth J. The importance of fluid balance in clinical practice. Nursing Standard 2009;22(47):50-57. [http://dx.doi.org/10.7748/ns2008.07.22.47.50.c6634]

3. Elliot D, Aitken L, Chaboyer W. ACCCN's Critical Care Nursing, 1st ed. Marrickville, Australia: Mosby Elsevier 2007:440,445-446.

4. Vincent JL, Sakr Y, Sprung CL, et al. Sepsis in European intensive care units: Results of the SOAP study. Crit Care intensive care units: Res

5. Alsous F, Khamiees M, DeGirolamo A, Amoateng-Adjepong $Y$, Manthous CA. Negative fluid balance predicts survival in patients with septic shock: A retrospective study. Chest 2000;117(6):1749-1754.

6. Boyd JH, Forbes J, Nakada T, Walley K, Russell JA. Fluid resuscitation in septic shock: A positive fluid balance and elevated central venous pressure are associated with increased mortality. Crit Care Med 2011;39(2):259-265. inttp://dx.doi.org/10.1097/CCM.0b013e3181feeb15]

7. Payen D, de Pont AC, Sakr Y, et al. A positive fluid balance Payen D, de Pont AC, Sakr Y, et al. A positive fluid balance
is associated with a worse outcome in patients with acute is associated with a worse outcome in patients with acut
renal failure. Crit Care 2008;12(3):R74. http://ccforum.com/ renal failure. Crit Care 2008;12(3):R74. http://ccforum.com/
content/12/3/R74 (accessed 27 October 2014). [http:// dx.doi.org/10.1186/cc6916]

8. Rosenberg AL, Dechert RE, Park PK, Bartlett RH, National Institutes of Health-National Heart, Lung, and Blood Institute Acute Respiratory Distress Syndrome Network. A review of a large clinical series: Association of cumulative fluid balance on outcome in acute lung injury: A retrospective review of the ARDSnet tidal volume study cohort. J Intensive Care Med 2009;24(1):35-46. [http:// dx.doi.org/10.1177/0885066608329850]

9. South African Nursing Council. R2598, Regulations relating to the scope of practice of persons who are registered or enrolled under the Nursing Act, 1978. Regulation of the Nursing Act, 2005 (Act No. 33 of 2005). Pretoria: Government Gazette, 491, 2006:34.

10. South African Government. Children's Act 38 of 2005 http://www.justice.gov.za/legislation/acts/2005-038\%20 childrensact.pdf (accessed 27 October 2014).

11. Perren A, Markmann M, Merlani G, Marone C, Merlani P. Fluid balance in critically ill patients. Should we really rely on it? Minerva Anestesiol 2011;77(8):802-811. 